\title{
Erratum to: Antimatroids and Balanced Pairs
}

\section{David Eppstein • Veit Wiechert}

Published online: 3 October 2014

(C) Springer Science+Business Media Dordrecht 2014

\section{Erratum to: Order (2014) 31(1):81-99 \\ DOI 10.1007/s11083-013-9289-1}

In the paper "Antimatroids and Balanced Pairs", published by the first author in Order 31(1):81-99, 2014, the proof of Lemma 12 is incorrect, but can be repaired, as was observed by V. Wiechert, with an argument similar to that in the incorrect proof.

Lemma 12 Let $x$ be an initial element of a set of orderings $\Omega$ that is preceded in relation $\prec$ by $i \geq 2$ other initial elements, and suppose that relation $\prec$ is total in $\Omega$. Then $\bar{r}(x)>$ $(2 i+5) / 3$.

Proof The expected rank of $x$ is one plus the expected number of elements of $\Omega$ that precede $x$ in a uniformly random ordering. If $S$ is the set of initial elements that precede $x$ in relation $\prec$, then the expected contribution of $S$ to the number of elements that precede $x$ in a random ordering is

$$
\sum_{y \in S} \operatorname{Pr}[y<x]>\frac{2 i}{3} .
$$

Adding the one unit for the fact that the rank is one even when there are zero elements preceding $x$, we obtain a total of $(2 i+3) / 3$.

To obtain the remaining $2 / 3$ of a unit in the expected rank given in the statement of the lemma, let $y$ be the immediate predecessor of $x$ in $\prec$, and let $z$ be the immediate predecessor

The online version of the original article can be found at http://dx.doi.org/10.1007/s11083-013-9289-1.

D. Eppstein $(\square)$

Computer Science Department, University of California, Irvine, CA, USA

e-mail: david.eppstein@gmail.com

V. Wiechert

Institute for Mathematics, Technical University of Berlin, Berlin, Germany 
of $y$ in $\prec$ (this is where we use the assumption that $i \geq 2$ ). Let $E_{1}$ be the event that $y$ precedes $x$ in an ordering and that at least one non-initial element occurs between $y$ and $x$ in the word. Let $E_{2}$ be the event that $x$ precedes $y$ and there is at least one non-initial element between them. Finally, let $E_{3}$ be the event that there are only initial elements between $x$ and $y$ in an ordering. Then $y$ precedes $x$ in all the orderings of $E_{1}$, none of the orderings of $E_{2}$, and half of the orderings in $E_{3}$. Since $y \prec x$, we obtain

$$
\operatorname{Pr}\left[E_{1}\right]+\frac{1}{2} \operatorname{Pr}\left[E_{3}\right]>\frac{2}{3} \text {. }
$$

Doubling this inequality and subtracting the equation $\operatorname{Pr}\left[E_{1}\right]+\operatorname{Pr}\left[E_{2}\right]+\operatorname{Pr}\left[E_{3}\right]=1$ (coming from the fact that these three events cover all orderings in $\Omega$ ) gives the equivalent form

$$
\operatorname{Pr}\left[E_{1}\right]-\operatorname{Pr}\left[E_{2}\right]>\frac{1}{3} \text {. }
$$

Now let $F$ be the event that $z$ precedes $y$ in an ordering and that at least one non-initial element occurs between $z$ and $y$ in the word. By similar analysis to the above we get $\operatorname{Pr}[F]>$ $1 / 3$. We split $F$ into the event $F^{-}$in which $x$ precedes the rightmost non-initial element between $y$ and $z$, and the event $F^{+}$in which $x$ does not precede this element; thus

$$
\operatorname{Pr}\left[F^{-}\right]+\operatorname{Pr}\left[F^{+}\right]>\frac{1}{3} \text {. }
$$

Because $F^{-}$is a subset of event $E_{2}$, we also have the inequality $\operatorname{Pr}\left[F^{-}\right] \leq \operatorname{Pr}\left[E_{2}\right]$.

Now let us count the non-initial predecessors of $x$. Whenever $E_{1}$ occurs we have a noninitial predecessor of $x$ that is a successor of $y$. Whenever $F^{+}$occurs we have a non-initial predecessor of $x$ that is not a successor of $y$. Thus, these two sets of non-initial predecessors are disjoint, and by linearity of expectation their expected number is

$$
\operatorname{Pr}\left[E_{1}\right]+\operatorname{Pr}\left[F^{+}\right]>\operatorname{Pr}\left[E_{1}\right]+\frac{1}{3}-\operatorname{Pr}\left[F^{-}\right] \geq \operatorname{Pr}\left[E_{1}\right]+\frac{1}{3}-\operatorname{Pr}\left[E_{2}\right]>\frac{2}{3} .
$$

Together with the contribution of the initial elements to the expected rank of $x$, this gives a lower bound of $(2 i+5) / 3$ on the expected rank, as stated. 